\section{Estrutura fatorial e consistência interna do Self-Reporting Questionnaire (SRQ-20) em população urbana}

\author{
Factor structure and internal consistency of the \\ Self-Reporting Questionnaire (SRQ-20) \\ in an urban population
}

\footnotetext{
${ }_{1}^{1}$ Departamento de Saúde, Universidade Estadual de Feira de Santana, Feira de Santana, Brasil.

Correspondência K. O. B. Santos Núcleo de Epidemiologia, Departamento de Saúde. Universidade Estadual de Feira de Santana.

Av. Universitária $s / n, \mathrm{Km} 03$ BR 116, VI Módulo, Campus Universitário, Feira de

Santana, $B A$

44031-460, Brasil.

kionnabernardes@hotmail.com
}

\begin{abstract}
This study focused on the psychometric properties of the Self-Reporting Questionnaire (SRQ-20) among workers, evaluating internal consistency criteria for the components scales and construct validity. Evaluation of the SRQ-20 performance used data from a cross-sectional study of a representative sample of workers from Feira de Santana, Bahia State, Brazil. The construct validity was estimated by means of an exploratory factor analysis of tetrachoric correlations and multiple correspondence analysis. The Kuder-Richardson formula (KD-20) was used to evaluate the instrument's internal consistency. Factor analysis of tetrachoric correlations revealed the extraction of four factors. Cumulative explanation of variance was 59.6\%. Multiple correspondence analysis identified categories according to the groupings of variables comprising the questionnaire. Overall and standardized internal consistency coefficient was 0.80. The study indicated an acceptable performance by the SRQ-20 for evaluating common mental disorders, for use in mental health screening in the workplace.
\end{abstract}

Questionnaires; Mental Health; Workers; Urban Population
Kionna Oliveira Bernardes Santos 1

Tânia Maria de Araújo ${ }^{1}$

Nelson Fernandes de Oliveira 1

A abordagem multicausal do adoecer, que leva em conta a interação entre fatores patogênicos, destacando a importância dos fatores ambientais, configura uma evolução dos métodos científicos para investigação da saúde 1 . Nesse âmbito, os estudos sobre estresse e a saúde mental dos trabalhadores têm ganhado relevo na comunidade científica.

Observa-se o crescimento acentuado de estudos voltados à avaliação dos aspectos relacionados à organização do trabalho e suas repercussões sobre a saúde do trabalhador 2. Porém, encontram-se limitações advindas das diversidades metodológica, teórica e conceitual, vistas nesses estudos.

Com o objetivo de avaliar os transtornos mentais comuns em países em desenvolvimento, a Organização Mundial da Saúde (OMS), orientada pela preocupação com os impactos que os problemas de saúde mental poderiam apresentar em países periféricos, desenvolveu o Self-Reporting Questionnaire (SRQ) ${ }^{3}$.

O SRQ avalia elementos relativos à saúde mental pertencentes a diferentes instrumentos para avaliação de transtornos mentais como: o General Health Questionnaire (GHQ-60), instrumento contendo sessenta questões; a versão reduzida do Present State Examination (PSE); o Post Graduate Institute Health Questionnaire N 2 (PGI), pesquisado na Índia; e o Patient Symptom Self Report (PASSR), instrumento desenvolvido na Colômbia 3 . 
A versão original do SRQ continha 24 itens, distribuídos em vinte questões para avaliação de transtornos não-psicóticos, e quatro para transtornos psicóticos como alucinações, delírio paranóide e confusão mental 4 .

Os transtornos mentais comuns, de acordo com Goldberg \& Huxley 5, caracterizam-se por sintomas não-psicóticos como: insônia, fadiga, irritabilidade, esquecimento, dificuldade de concentração e queixas somáticas.

OSRQ tornou-se um instrumento amplamente utilizado para suspeição diagnóstica dos transtornosmentaiscomuns; naversãobrasileiraforam retiradas as quatro questões referentes aos distúrbios psicóticos mantendo-se a versão com vinte itens ${ }^{3}$. O SRQ já foi traduzido para oito idiomas diferentes, sendo utilizado em vários países como: Colômbia, Índia, Filipinas, Sudão 6, Etiópia 7, Guiné-Bissau ${ }^{8}$, Quênia 9 , Brasil ${ }^{3}$ e Senegal 10.

Na década de 90, o SRQ-20 destacou-se como o instrumento de triagem de morbidade psíquica mais utilizado nos estudos brasileiros 11,12,13. Entretanto, apesar do SRQ-20 ser amplamente utilizado, sua validação restringiu-se a poucos estudos que avaliaram populações provenientes de serviços de atenção primária ${ }^{3}$ e de duas categorias profissionais específicas 13,14.

Este estudo tem como objetivo investigar as propriedades psicométricas do SRQ-20, entre uma população urbana de trabalhadores, avaliando critérios de consistência interna das escalas componentes e a validade de construto.

A avaliação do desempenho dos instrumentos de pesquisa usados é de fundamental importância para se precisar, criteriosamente, a fidedignidade dos aspectos investigados, especialmente quando se considera um instrumento como o SRQ-20 que foi desenvolvido para uso em serviços de atenção primária em saúde, portanto, para a elucidação de contextos diversos das situações comumente encontradas em ambientes laborais.

O processo de mensuração nas ciências psicossociais é realizado utilizando-se instrumentos de mensuração e/ou medição representados por um teste, uma escala ou um questionário ${ }^{15}$. Quando se aplica um instrumento para mensuração de um evento com múltiplas dimensões como a saúde mental, a avaliação criteriosa de seu desempenho, ou seja, a validação deste instrumento, deve ser realizada 16 .

Para Streiner \& Norman 17, a validade de um instrumento é observada após a aplicação de um conjunto de técnicas que tem por objetivo avaliar o grau de significância real de um conceito representado por uma medida empírica.

No entanto, para ser considerado válido um instrumento também deve ser confiável sendo a confiabilidade necessária, mas não suficiente para condição de validade. A relação existente entre validade e confiabilidade pode ser analisada por meio da consistência presente em critérios externos (validez) e critérios internos (fidedignidade) 18 .

A consistência interna se refere à inter-relação de um grupo de itens que compõe um instrumento de investigação que representa uma condição necessária para a homogeneidade das mensurações, porém não suficiente para assegurar a unidimensionalidade do teste 19 .

Este estudo se insere nos esforços para o adequado dimensionamento de fatores envolvidos na saúde mental, objetivando avançar na avaliação do SRQ-20 ao investigar aspectos da validade de construto e confiabilidade dos itens que compõem este instrumento.

\section{Metodologia}

Para avaliação do desempenho do SRQ-20 foi utilizado o banco de dados disponibilizado pelo Núcleo de Epidemiologia do Departamento de Saúde, da Universidade Estadual de Feira de Santana, referente a um estudo intitulado Distúrbios Psíquicos Menores: Estimativas de Prevalência e Validação de Instrumento Diagnóstico Self-Reporting Questionnaire (SRQ-20). Esta pesquisa foi aprovada pelo Comitê de Ética em Pesquisa com Seres Humanos do Hospital São Rafael, sob protocolo 17/01, atendendo aos requisitos da Resolução $n^{\circ}$. 196/96 do Conselho Nacional de Saúde.

Um estudo de corte transversal foi realizado numa amostra representativa da população urbana de Feira de Santana, Bahia, Brasil. A amostra foi composta por 3.190 indivíduos com idades de 15 anos ou mais, selecionados por meio de amostragem aleatória por conglomerado. Para o cálculo do tamanho da amostra se assumiu uma prevalência estimada de transtornos mentais de $24 \% 20$, erro amostral de $3 \%$ e $95 \%$ de confiança. Para correção do efeito do desenho de estudo dobrou-se o tamanho da amostra. A população de trabalhadores ativos avaliados no momento da pesquisa foi de 1.311, distribuídos entre os setores informal e formal.

O SRQ-20, instrumento multidimensional, foi utilizado para a suspeição diagnóstica de transtornos mentais comuns. Na Tabela 1 estão contidos os itens do SRQ-20.

A validade de construto foi estimada por meio da análise fatorial tetracórica e pela análise de correspondência múltipla. A fórmula de KuderRichardson (KD-20) foi empregada para avaliar a consistência interna do instrumento, utilizan- 
Itens do Self-Reporting Questionnaire (SRQ-20) distribuídos por quatro grupos de sintomas.

\begin{tabular}{|c|c|}
\hline Grupo de sintomas & Questões do SRQ-20 \\
\hline \multirow[t]{4}{*}{ Humor depressivo-ansioso } & Sente-se nervoso, tenso ou preocupado? \\
\hline & Assusta-se com facilidade? \\
\hline & Sente-se triste ultimamente? \\
\hline & Você chora mais do que de costume? \\
\hline \multirow[t]{6}{*}{ Sintomas somáticos } & Tem dores de cabeça freqüentemente? \\
\hline & Você dorme mal? \\
\hline & Você sente desconforto estomacal? \\
\hline & Você tem má digestão? \\
\hline & Você tem falta de apetite? \\
\hline & Tem tremores nas mãos? \\
\hline \multirow[t]{6}{*}{ Decréscimo de energia vital } & Você se cansa-se com facilidade? \\
\hline & Tem dificuldade em tomar decisão? \\
\hline & Tem dificuldades de ter satisfação em suas tarefas? \\
\hline & O seu trabalho traz sofrimento? \\
\hline & Sente-se cansado todo o tempo? \\
\hline & Tem dificuldade de pensar claramente? \\
\hline \multirow[t]{4}{*}{ Pensamentos depressivos } & Sente-se incapaz de desempenhar papel útil em sua vida? \\
\hline & Tem perdido o interesse pelas coisas? \\
\hline & Tem pensado em dar fim à sua vida? \\
\hline & Sente-se inútil em sua vida? \\
\hline
\end{tabular}

do-se como parâmetro os valores de referência indicados na literatura $0,65-0,9021$.

\section{Análise fatorial tetracórica}

A identificação de fatores entre os itens que compõem o questionário foi realizada por meio da análise fatorial de correlações tetracóricas. Incluiu-se nesses a população trabalhadora $(\mathrm{n}=1.311)$.

A opção por essa técnica justifica-se por ser a mais apropriada para avaliar variáveis dicotômicas e pelo estudo possuir um grande número amostral, o que minimiza o risco da ocorrência de alto de erro padrão dos coeficientes de correlação tetracórica ${ }^{25}$. Sendo, portanto, indicada para avaliar a relação entre um grupo de variáveis, permitindo o agrupamento de itens que mantenham relação entre si.

Inicialmente foi feita uma análise exploratória para todos os itens do questionário, seguida da extração de fatores utilizando-se a rotação ortogonal Varimax. Segundo Kline 23, essa técnica permite maior facilidade de interpretação dos fatores extraídos. Para essa análise, os itens que apresentaram carga superior a 0,40 foram considerados como os mais importantes na composição dos fatores.
Para a análise dos dados foi utilizado o programa Stata na versão 9.0 (Stata Corp., College Station, Estados Unidos).

\section{Análise de correspondências múltiplas}

Análise de correspondência é uma técnica descritiva gráfica para a análise de dados categorizados, que facilita a interpretação das relações entre as variáveis envolvidas. Ela é especialmente apropriada para a avaliação de tabelas de duas variáveis, mas pode ser estendida para a análise de tabelas com mais de duas variáveis, em que as complexas associações são apresentadas usualmente num gráfico de duas dimensões, de tal modo que não haja grande perda de informação.

As variáveis foram rotuladas assumindo-se os seguintes valores: 1 (sim) e 2 (não). Foram considerados os dois primeiros eixos para interpretação: o primeiro, Eixo 1, apresenta a separação entre as respostas positivas e negativas; o segundo, Eixo 2, representa a distância entre as variáveis com respostas positivas.

A técnica consiste, essencialmente, na projeção de pontos de um espaço multidimensional para o plano bidimensional usando-se um critério semelhante ao de mínimos quadrados, baseado numa medida de distância entre os pontos, 
conhecida como "a distância qui-quadrado" 24. Procura-se explicar o máximo da variabilidade dos dados (medida pela distância qui-quadrado) por meio da projeção dos pontos no plano bidimensional, o que permite a visualização de padrões de "proximidade" entre os pontos e a interpretação das associações entre as variáveis.

Desse modo, o objetivo principal da análise de correspondência é reduzir um conjunto de informações para uma representação gráfica em dois eixos, ao estabelecer relações entre grupos de variáveis e permitir uma representação simplificada das relações simultâneas 25 .

Para essa análise foi utilizado o programa R versão 2.2.1 (The R Foundation for Statistical Computing, Viena, Áustria; http://www.rproject.org).

\section{Resultados}

O perfil da população de trabalhadores revelou proporções similares segundo sexo: $49,7 \%$ eram do sexo feminino e $50,3 \%$ do masculino; a média de idade foi de 35,1 ( $\pm 13,3)$ anos; 7,9\% não possuíam nenhum grau de instrução, enquanto 43,2\% tinham Ensino Fundamental e 48,4\% nível superior.

A análise fatorial de correlações tetracóricas extraiu quatro fatores que compunham dimensões específicas, identificadas como: fator I - comportamento ansioso e depressivo, fator II - decréscimo de energia, fator III - sintomas somáticos, fator IV - humor depressivo. Entre os fatores identificados na análise, o IV e o III apresentaram maior clareza para interpretação (Tabela 2).

A proporção cumulativa de explicação da variância entre os trabalhadores estudados foi de $59,6 \%$, indicando que os quatro fatores extraídos pela análise fatorial de correlações tetracóricas possuem um bom nível de explicação para a proporção de variância do SRQ-20.

As questões "Tem chorado mais que o costume?" e "Sente-se triste ultimamente?", destacaram-se por possuir as maiores cargas: 0,8206 e 0,7353 , respectivamente, na extração do fator I, que, após rotação, apresentou a maior proporção de variância explicada, 17,13\%.

Quatro questões se agruparam para a formação do fator II, discriminado como "decréscimo de energia". Nesse fator foram identificadas as maiores cargas nas questões "O seu trabalho traz sofrimento?", e "Tem dificuldade de ter satisfação em suas tarefas?"

O fator III apresentou cargas maiores que 0,40 para quatro questões relacionadas aos sintomas somáticos. Dentre elas as que se agruparam para a formação desse fator destacaram-se, por possuir maior carga, as questões relacionadas à má digestão e a sensações desconfortáveis no estômago, 0,8396 e 0,8878, respectivamente. As outras duas questões extraídas nesse fator foram: "Você tem falta de apetite?" e "Você tem dores de cabeça freqüentemente?”.

$\mathrm{O}$ fator IV apresentou o agrupamento de seis questões que descrevem sintomas característicos de humor depressivo, como a dificuldade de tomar decisão, falta de interesse, sensação de inutilidade, dificuldade de sentir satisfação, dificuldade de pensar claramente e idéias de acabar com a vida.

Por meio da análise de correspondências múltiplas foi possível identificar agrupamentos de acordo com o grau de proximidade entre as categorias das variáveis que compunham o questionário.

A interpretação foi restrita ao Eixo 2, que representa a distância existente entre as variáveis com respostas positivas.

A Figura 1 mostra a proximidade entre as variáveis sugerindo agrupamentos de acordo com as distâncias existentes entre as categorias. Em geral, observou-se atração entre as variáveis do SRQ-20, identificando a formação de quatro grupos. Destacaram-se as variáveis "es" (“Tem sensações desagradáveis no estômago?”) e "md" ("Você tem má digestão?"), no quadrante superior esquerdo e no extremo oposto um grupo de variáveis que mantinham uma relação de proximidade "fi" ("Tem tido idéia de acabar com a vida?"), "si" ("Tem perdido o interesse pelas coisas?"), "iv" ("Você é incapaz de desempenhar um papel útil em sua vida?"), "in” ("Você se sente pessoa inútil em sua vida?”). As outras variáveis formaram dois grupos próximos à linha central, representados no quadrante superior esquerdo por "ct" ("Sente-se cansado(a) o tempo todo?"), "tb" ("Seu trabalho diário lhe causa sofrimento?"), "sa" ("Encontra dificuldade de realizar, com satisfação, suas tarefas diárias?”), "sf” ("Tem falta de apetite?"), "dm" ("Você dorme mal?"), "cs" ("Você se cansa com facilidade?"), "cb" ("Tem dores de cabeça freqüentemente?"), "su” ("Assusta-se com facilidade?"), "ts" ("Sente-se nervoso(a), tenso(a) ou preocupado(a)?”) e, no quadrante inferior esquerdo "tr" ("Tem tremores nas mãos?"), "ch" ("Tem chorado mais do que de costume?"), "tt" ("Tem se sentido triste ultimamente?”), "ps” ("Tem dificuldade de pensar com clareza?"), "dc" ("Tem dificuldade para tomar decisões?").

A consistência interna identificada dos itens que compõem o SRQ-20 foi avaliada globalmente e por estratos ao se considerar diferenças relativas ao sexo e escolaridade dos trabalhadores (Tabela 3). 
Classificação dos fatores encontrados na aplicação do Self-Reporting Questionnaire (SRQ-20) na população de trabalhadores de Feira de Santana, Bahia, Brasil, 2001

\begin{tabular}{|c|c|c|c|c|}
\hline \multirow[t]{2}{*}{ Questões do SRQ-20 } & \multicolumn{4}{|c|}{ Trabalhadores } \\
\hline & Fator I & Fator II & Fator III & Fator IV \\
\hline \multicolumn{5}{|l|}{ Humor depressivo-ansioso } \\
\hline Sente-se triste ultimamente? & 0,7353 & - & - & - \\
\hline Você dorme mal? & 0,4709 & - & - & - \\
\hline Você chora mais que de costume? & 0,8206 & - & - & - \\
\hline Sente-se nervoso, tenso, preocupado? & 0,5303 & - & - & - \\
\hline Tem tremores nas mãos? & 0,4369 & - & - & - \\
\hline Assusta-se com facilidade? & 0,4506 & - & - & - \\
\hline \multicolumn{5}{|l|}{ Decréscimo de energia vital } \\
\hline O seu trabalho traz sofrimento? & - & 0,8484 & - & - \\
\hline Você se cansa com facilidade? & - & 0,5970 & - & - \\
\hline Sente-se cansado todo o tempo? & - & 0,5953 & - & - \\
\hline Tem dificuldade de ter satisfação em suas tarefas? & - & 0,8079 & - & - \\
\hline \multicolumn{5}{|l|}{ Sintomas somáticos } \\
\hline Você sente desconforto estomacal? & - & - & 0,8878 & - \\
\hline Você tem falta de apetite? & - & - & 0,4992 & - \\
\hline Você tem má digestão? & - & - & 0,8396 & - \\
\hline Tem dores de cabeça freqüentemente? & - & - & 0,4291 & - \\
\hline \multicolumn{5}{|l|}{ Pensamentos depressivos } \\
\hline Tem dificuldade de tomar decisão? & - & - & - & 0,6810 \\
\hline Tem perdido interesse pelas coisas? & - & - & - & 0,6517 \\
\hline Sente-se inútil em sua vida? & - & - & - & 0,6776 \\
\hline Tem dificuldade de pensar claramente? & - & - & - & 0,5886 \\
\hline Sente-se incapaz de desempenhar papel útil em sua vida? & - & - & - & 0,6919 \\
\hline Tem pensado em dar fim à sua vida? & - & - & - & 0,4739 \\
\hline \multicolumn{5}{|l|}{ Total } \\
\hline Variância explicada após rotação (\%) & 17,13 & 15,52 & 12,86 & 14,05 \\
\hline Total de variância explicada (\%) & 59,6 & - & - & - \\
\hline
\end{tabular}

O coeficiente de consistência interna geral e padronizado foi de 0,80 ; após o agrupamento dos itens por grupos de sintomas, feito pela análise fatorial tetracórica, procedeu-se a análise da consistência interna dos grupos obtidos. Os coeficientes encontrados variaram de 0,57 a 0,65 revelando desempenho regular. A consistência interna mais elevada foi observada para o grupo de variáveis que compunham o fator comportamento ansioso/depressivo $(\alpha=0,65)$, seguida por sintomas somáticos $(\alpha=0,62)$, entretanto as duas outras dimensões, humor depressivo $(\alpha=0,59) \mathrm{e}$ decréscimo de energia $(\alpha=0,57)$ apresentaram coeficientes mais baixos.

Os melhores coeficientes foram encontrados entre os trabalhadores que possuíam menor grau de instrução (< 5 anos), variando entre 0,59 e 0,68 e coeficiente global de $\alpha=0,82$. Entre as trabalhadoras o fator comportamento ansioso/depressivo revelou o maior coeficiente $(\alpha=0,66)$, e o pior coeficiente foi identificado no fator sintomas somáticos $(\alpha=0,59)$.

Entre os homens foram identificados os menores valores para os coeficientes de consistência interna que variaram entre 0,52 e 0,57 , entretanto, quando os itens foram avaliados globalmente o coeficiente encontrado $(\alpha=0,75)$ apresentouse segundo os parâmetros indicados na literatura 22. Desempenho semelhante foi identificado entre os trabalhadores que possuíam maior grau de instrução ( $\geq 5$ anos).

\section{Discussão}

Apesar de alguns estudos utilizarem a técnica tradicional de análise fatorial convencional para verificar o desempenho do SRQ-20 4,22, este estudo optou pela análise fatorial exploratória de correlações tetracóricas como um dos mé- 
Visualização da proximidade entre as variáveis do Self-Reporting Questionnaire (SRQ-20), de acordo com a análise de correspondências múltiplas.

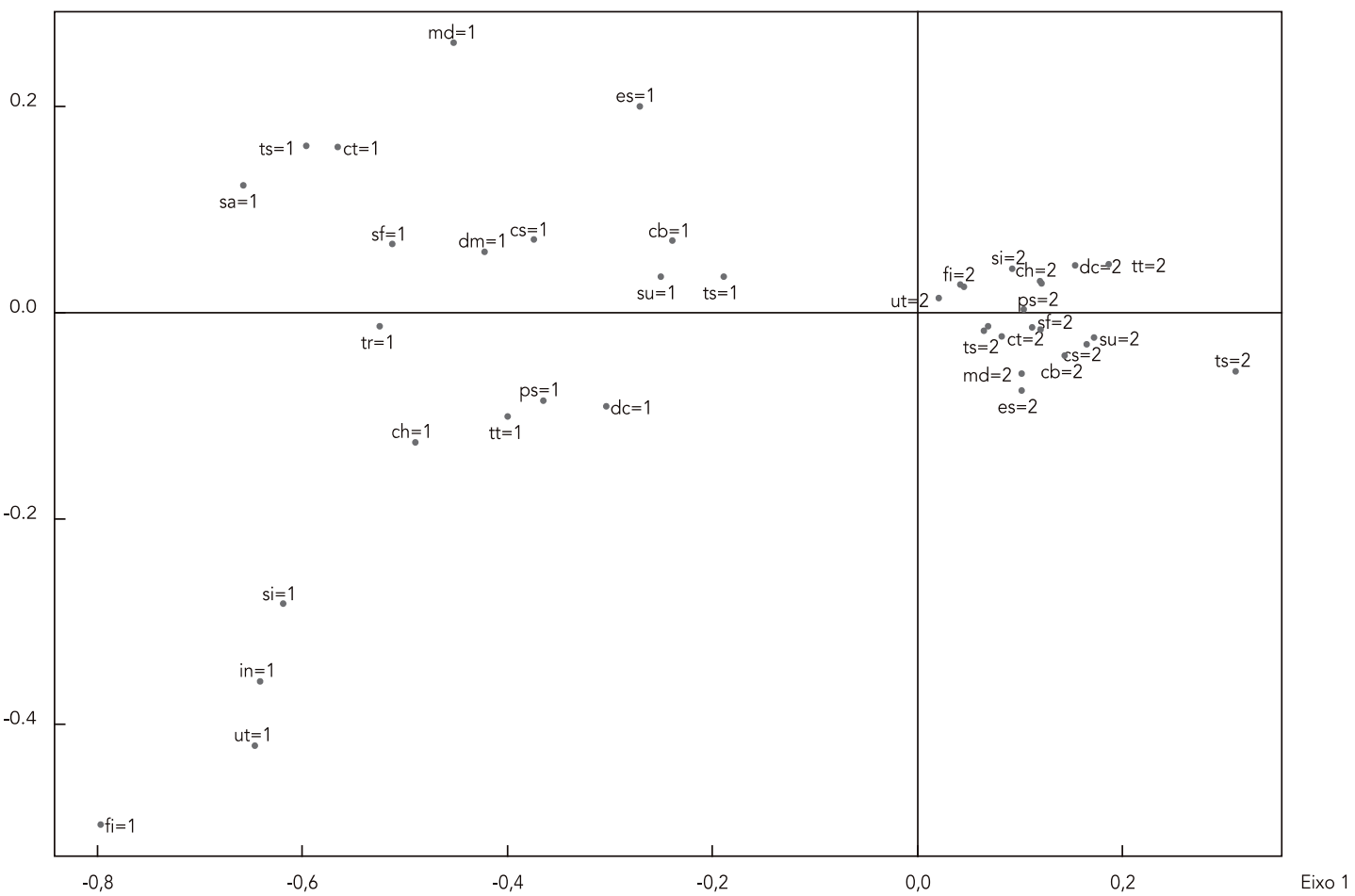

Tabela 3

Itens do Self-Reporting Questionnaire (SRQ-20) distribuídos por quatro grupos de sintomas.

\begin{tabular}{|c|c|c|c|c|c|}
\hline Fatores & Trabalhadores & Homens & Mulheres & $\begin{array}{l}\text { Escolaridade } \\
\text { ( }<5 \text { anos })\end{array}$ & $\begin{array}{c}\text { Escolaridade } \\
\text { ( } \geq 5 \text { anos) }\end{array}$ \\
\hline Comportamento ansioso e depressivo (fator I) & 0,65 & 0,57 & 0,66 & 0,68 & 0,58 \\
\hline Decréscimo de energia (fator II) & 0,57 & 0,54 & 0,65 & 0,64 & 0,57 \\
\hline Sintomas somáticos (fator III) & 0,62 & 0,55 & 0,59 & 0,59 & 0,53 \\
\hline Humor depressivo (fator IV) & 0,59 & 0,52 & 0,61 & 0,62 & 0,48 \\
\hline Global & 0,80 & 0,75 & 0,80 & 0,82 & 0,74 \\
\hline
\end{tabular}

todos aplicados para validação de construto do SRQ-20. Os resultados demonstraram habilidade dessa técnica na extração dos fatores e na interpretação dos dados que discriminaram aspectos específicos na mensuração das dimensões dos transtornos mentais avaliadas no SRQ-20.

Foi verificada, também, uma melhor explicação da proporção de variância ao se utilizar a análise fatorial exploratória de correlações tetracóricas apontando, desta forma, resultados mais consistentes para explicação da proporção de variância explicada, superior aos encontrados em outros estudos que utilizaram a técnica de análise fatorial convencional 4,22 .

Entre os trabalhadores, os fatores referentes ao "decréscimo de energia" e "sintomas somá- 
ticos" apresentaram itens com altos valores de carga, revelando que estes sintomas são os mais adequadamente mensurados nos grupos ocupacionais pesquisados.

Este estudo verificou similaridade entre o fator I, denominado "comportamento ansioso depressivo" e o fator IV relativo ao "humor depressivo". Nesses dois fatores não foi possível identificar claramente manifestações distintas dos sintomas na população de trabalhadores estudada, concordando assim com Iacoponi \& Mari ${ }^{4}$ que afirmam que as subescalas (questões) que compunham esses dois fatores indicavam problemas de interpretação.

O fator III "sintomas somáticos" se destacou facilmente dos demais. Esse achado concorda com os resultados apontados na literatura que também relataram a discriminação clara desse fator 4,22

A análise fatorial exploratória apesar de ter apresentado a extração de quatro fatores, neste estudo não foi realizado o teste de hipótese para a confirmação e o refinamento dos resultados. Dessa forma, torna-se necessário um melhor detalhamento por meio da análise fatorial confirmatória que permita estabelecer comparações entre modelos previamente testados.

Outro método empregado na validação de construto do SRQ-20 foi a análise de correspondências múltiplas. Correspondências foram encontradas entre as variáveis, avaliadas pela "proximidade" existente entre elas. A explicação da inércia total de acordo com a análise de correspondências múltiplas indicou uma representação gráfica das relações entre as variáveis de qualidade satisfatória.

A alocação de pontos no espaço, conduzida na análise de correspondências múltiplas, usando-se o agrupamento de variáveis, fortaleceu a classificação dos quatro fatores sugeridos pela análise fatorial tetracórica, ao mesmo tempo em que revelou dimensões extremas (sintomas somáticos/humor depressivo), admitindo-se que quanto mais distantes encontram-se as variáveis, menos freqüente será a sua ocorrência em conjunto. Esse achado expressa a natureza multidimensional dos transtornos mentais, avaliados pelo SRQ-20, concordando assim com Iacoponi \& Mari 4.

É necessário destacar, entretanto, a fragilidade da análise de correspondência múltipla para o real dimensionamento de questões subjetivas como os transtornos mentais comuns.

Outra característica observada foi a proximidade encontrada entre as variáveis que compu- nham os fatores relacionados a "decréscimo de energia" e "comportamento ansioso depressivo", o que denota a não diferenciação dos sintomas envolvidos para a manifestação dos transtornos mentais comuns com relação a estes sintomas, já relatada por Shepherd 26

Ao se avaliar a consistência interna do SRQ20, o resultado encontrado neste estudo concorda com o registrado na literatura 4 , em que o valor de um único coeficiente $(\alpha=0,81)$ foi descrito como referência para avaliar as vinte questões que compõem o instrumento, atestando uma alta correlação entre as questões.

Neste estudo, a consistência interna do SRQ20 foi avaliada também usando-se os fatores (itens) extraídos após a análise fatorial tetracórica, bem como estratos diferenciados por sexo e escolaridade. De maneira geral, os coeficientes de consistência interna entre as escalas do SRQ20 apresentaram valores mais baixos que os observados como referência na literatura 18 .

Cherian et al. 22, ao avaliarem uma população de estudantes de Ensino Médio na África do Sul, encontraram coeficientes de consistência interna entre as escalas do SRQ-20 com altos valores $(\alpha=0,89)$ para os quatro fatores extraídos por meio de análise fatorial convencional. Entretanto, no presente estudo, entre os trabalhadores que possuíam maior grau de instrução foram identificados os menores valores de coeficientes de consistência interna quando se avaliou o grupo de sintomas extraídos pela análise fatorial tetracórica.

Uma discussão tem sido fomentada na literatura para a interpretação dos baixos valores dos coeficientes de consistência interna do SRQ-20 quando se considera separadamente os grupos de sintomas. A justificativa encontrada para os baixos valores repousa sobre o conceito de que os transtornos mentais não possuem características unidimensionais e que, portanto, não estabelecem relações homogêneas entre os itens que compõem as suas escalas 20. Dessa forma, quando os itens são avaliados globalmente o indicador geral de consistência interna do instrumento apresenta resultado satisfatório.

Este estudo aponta para um desempenho aceitável do SRQ-20 em avaliar os transtornos mentais comuns, ao destacar que, apesar da natureza múltipla dos transtornos emocionais, o instrumento demonstrou habilidade em identificar fatores que, juntos, denotam características indispensáveis para o rastreamento da saúde mental em âmbito ocupacional. 


\section{Resumo}

Este estudo teve como objetivo investigar as propriedades psicométricas do Self-Reporting Questionnaire (SRQ-20) entre trabalhadores, avaliando critérios de consistência interna das escalas componentes e a validade de construto. Para avaliação do desempenho do SRQ-20 foram utilizados dados de um estudo de corte transversal realizado numa amostra representativa dos trabalhadores de Feira de Santana, Bahia, Brasil. A validade de construto foi estimada por meio da análise fatorial exploratória de correlações tetracóricas e pela análise de correspondência múltipla. A fórmula de Kuder-Richardson (KD-20) foi empregada para avaliar a consistência interna do instrumento. A análise fatorial de correlações tetracóricas revelou a extração de quatro fatores. A proporção cumulativa de explicação da variância foi de 59,6\%. Utilizando-se a análise de correspondências múltiplas foi possível identificar categorias de acordo com os agrupamentos das variáveis que compunham o questionário. O coeficiente de consistência interna geral e padronizado foi de 0,80. Este estudo aponta para um desempenho aceitável do SRQ-20 em avaliar os transtornos mentais comuns, com rastreamento da saúde mental em âmbito ocupacional.

Questionários; Saúde Mental; Trabalhadores; População Urbana

\section{Colaboradores}

K. O. B. Santos participou na elaboração, análise de dados e revisão do artigo. T. M. Araújo colaborou na orientação, elaboração e revisão do artigo. N. F. Oliveira contribuiu na consultoria estatística, elaboração e revisão do artigo.

\section{Agradecimentos}

Projeto subvencionado pelas agências financiadoras: Coordenação de Aperfeiçoamento de Pessoal de Nível Superior (CAPES; bolsa) e Fundação de Amparo à Pesquisa do Estado da Bahia (FAPESB; auxílio dissertação $n^{\circ}$. de convênio 4183).

\section{Referências}

1. Albrecht KG. Stress and the manager. New Jersey: Prentice Hall; 1979.

2. Seligmann-Silva E. Desgaste mental no trabalho dominado. Rio de Janeiro: Editora da Universidade Federal do Rio de Janeiro/São Paulo: Cortez Editora; 1994.

3. Mari JJ, Williams P. A validity study of a psychiatric screening questionnaire (SRQ-20) in primary care in the city of São Paulo. Br J Psychiatry 1986; 148:23-6.
4. Iacoponi E, Mari JJ. Reliability and factor structure of the Portuguese version of Self-Reporting Questionnaire. Int J Soc Psychiatry 1998; 35:213-22.

5. Goldberg D, Huxley P. Common mental disorders: a bio-social model. London: Tavistock; 1992.

6. Harding TW, Arango MV, Baltazar J, Climent CE, Ibrahim HH, Ladrido-Ignacio L, et al. Mental disorders in primary health care: a study of their frequency and diagnosis in four developing countries. Psychol Med 1980; 10:231-41. 
7. Kortmann F. Problems in communication in transcultural psychiatry. The self reporting questionnaire in Ethiopia. Acta Psychiatr Scand 1987; 75:563-70.

8. de Jong JT, de Lein GA, ten Horn SG. A baseline study on mental disorders in Guiné-Bissau. Br J Psychiatry 1986; 148:27-32.

9. Dhadphale R, Ellison RH, Griffin L. The frequency of psychiatric disorders among patients attending semi-urban and rural general out-patient clinics in Kenya. Br J Psychiatry 1983; 142:379-83.

10. Diop B, Collignon R, Guèye M, Harding TW. Diagnosis and symptoms of mental disorder in a rural area of Senegal. Afr J Med Med Sci 1982; 11:95-103.

11. Araújo TM. Trabalho e distúrbios psíquicos em mulheres trabalhadoras de enfermagem [Tese de Doutorado]. Salvador: Instituto de Saúde Coletiva, Universidade Federal da Bahia; 1999.

12. Borges LH, Faria MAM. Transtornos mentais menores entre trabalhadores de uma usina siderúrgica. Rev Bras Saúde Ocup 1993; 21:7-18.

13. Palácios M, Jardim S, Ramos A, Silva Filho JF. Validação do Self-Report Questionnaire-20 (SRQ-20) numa população de trabalhadores de um banco estatal no Rio de Janeiro, Brasil. In: Silva Filho JF, Jardim S, organizadores. A danação do trabalho: organização do trabalho e sofrimento psíquico. Rio de Janeiro: Te Corá Editora; 1998. p. 225-41.

14. Fernandes SRP, Almeida Filho N. Validação do SRQ-20 em amostra de trabalhadores de informática. Rev Bras Saúde Ocup 1997; 24:105-12.

15. Souza J, Penalosa RAS. Mensuração. http://www. ufrgs.br/ppge/pcientifica/2005_02.pdf (acessado em 15/Out/2005).

16. Fletcher R, Fletcher S, Wagner E. Epidemiologia clínica: elementos essenciais. 3a Ed. Porto Alegre: Editora Artes Médicas; 1996.
17. Streiner DL, Norman GR. Health measurement scales: a practical guide to their development and use. 2nd Ed. Oxford: Oxford University Press; 1995.

18. Richardson RJ, Peres JAS, Wanderley JCV, Correia LM, Peres MHM. Pesquisa social: métodos e técnicas. 3a Ed. São Paulo: Editora Atlas; 1999.

19. Schmidt FL, Hunter JE. Measurement error in psychological research: lessons from 26 research scenarios. Psychol Methods 1996; 1:199-223.

20. Organização Mundial da Saúde/Organização PanAmericana da Saúde. Relatório sobre a saúde no mundo. Saúde mental: nova concepção, nova esperança. Genebra: Organização Mundial da Saúde; 2001.

21. Nunnaly J. Psychometric theory. New York: McGraw-Hill; 1978

22. Cherian VI, Peltzer K, Cherian L. The factor-structure of the Self Reporting Questionnaire (SRQ-20) in South Africa. East Afr Med J 1998; 75:654-6.

23. Kline P. An easy guide to factors analysis. London: Routledge; 1994.

24. Greenacre MJ. Correspondence analysis in practice. London: Academic Press; 1993.

25. Aranha RN, Faerstein E, Azevedo GM, Werneck G Lopes CS. Análise de correspondência para avaliação do perfil de mulheres na pós-menopausa e o uso da terapia de reposição hormonal. Cad Saúde Pública 2004; 20:100-8.

26. Shephered M. The extent of mental disease beyond Layman's madness. In: Tanner, JM, editor. Developments in psychiatry research. London: Hodder \& Stoughton; 1977. p. 178-98.

Recebido em 25/Jul/2007

Versão final reapresentada em 09/Mai/2008

Aprovado em 20/Mai/2008 